\title{
A GENERALIZED PROBABILISTIC ANALYSIS OF A POLYHEDRON'S NUMBER OF SHADOW VERTICES
}

\author{
Karl-Heinz Küfer, Universität Kaiserslautern
}

Introduction: Let $A:=\left\{a_{1}, \ldots, a_{m}\right\}, a_{i} \in \mathbb{R}^{\mathbf{n}}, m \geq n \geq 2$, where vectors $a_{i}$ are considered i.i.d.-vectors under an arbitrary rotationally symmetric distribution in $\mathbb{R}^{\mathbf{n}}$. The random event $A$ may be identified with a polyhedron $X_{A}$ by $X_{A}:=\left\{x \in \mathbb{R}^{\mathbf{n}} \mid a^{T} x \leq 1, a \in A\right\}$. Furthermore, let $L_{k}, L_{k}:=\operatorname{span}\left(u_{1}, \ldots, u_{k}\right), u_{i} \in S^{n-1}$, be an arbitrary $\mathbb{R}^{\mathbf{n}}$-subspace of dimension $k \in\{1, \ldots, n\}$. We call a vertex of $X_{A}$ " $L_{k}$-shadow vertex" if it remains a vertex of $X_{A}$ 's image under an orthogonal projection into $L_{k}$. The number of $X_{A}$ 's $L_{k}$-shadow vertices will be denoted by $S_{L_{k}}\left(X_{A}\right)$. Knowledge about a polyhedron's number of shadow vertices is of interest within the probabilistic analysis of algorithms, where the the cardinality of a polyhedron's vertex subset is the main benchmark of complexity. A famous example is the "shadow vertex algorithm" - a variant of the simplex algorithm - in case of planary projection spaces $(k=2)$, cf. $[1,2]$.

Results: We study expectation value and variance of the random variable $S_{L_{k}}\left(X_{A}\right)$ in two different ways, the first leading to asymptotic results for fixed dimension $n, m \rightarrow \infty$, and special distributions, the latter overestimating moments independently from the underlying distribution. In both cases we concentrate on the increasing rate in $m$ and give only qualitative results about the growth in $n, k$ is always assumed to be fixed.

In case of expectation values a typical asymptotic result among many others is the following:

$$
\mathrm{E}\left(S_{L_{k}}\right)=C_{n, k} m^{(k-1) /(n+1)}(1+o(1)), m \rightarrow \infty,
$$

in case of $a_{i}$ being uniformly distributed in the unit ball of $\mathbb{R}^{\mathbf{n}}, n \geq 2$. Here, the constant $C_{n, k}$ is polynomial in $n$ for fixed $k$. Independently from the underlying rotationally invariant distribution holds:

$$
\mathrm{E}\left(S_{L_{k}}\right) \leq C_{n, k} m^{(k-1) /(n-1)}, m \geq n \geq 2,
$$

$C_{n, k}$ being polynomial in $n$ for fixed $k$ again. Corresponding results for variances of $S_{L_{k}}$ are

$$
\frac{\operatorname{Var}\left(S_{L_{k}}\right)}{\mathrm{E}^{2}\left(S_{L_{k}}\right)}=\Theta\left(m^{-(k-1) /(n+1)}\right), m \rightarrow \infty .
$$

for uniformly distributed $a_{i}$ in the $n$-dimensional unit ball and

$$
\frac{\operatorname{Var}\left(S_{L_{k}}\right)}{\mathrm{E}^{2}\left(S_{L_{k}}\right)} \leq C_{n, k}
$$

which holds independently from the underlying distribution. In (3) and (4) the constants $C_{n, k}$ are polynomial in $n$ for fixed $k$. By the aid of Chebychev's inequality (3) implies that in case of large $m$ and fixed $n$ even small relative deviations from the mean are very rare. This observation is true for all rotationally invariant distributions with compact support, while there are distributions with non-compact support, where the investigated quotient in (3) and (4) is positively bounded from below.

\section{References:}

[1] Borgwardt, K. H. (1982) The Average Number of Pivot Steps Required by The Simplex Method is Polynomial. ZOR 26: 157-177.

[2] Küfer, K. H. (1992) On the Variance of the Number of Pivot Steps Required by the Simplex Algorithm. To appear in ZOR, Preprint Universität Kaiserslautern. 